\title{
A Review of the Therapeutic Interventions in the Management of Pruritus Ani
}

\author{
Arifa Siddika*, Shahab Siddiqi \\ Department of Surgery (Colorectal), Broomfield Hospital, Mid Essex NHS Trust, Chelmsford, UK
}

*Corresponding author: Ms. Arifa Siddika, Registrar- Colorectal surgery, Broomfield Hospital, Mid Essex NHS Trust, Chelmsford, Essex, CM1 7ET, UK; E-mail: drsiddika@yahoo.com

\begin{abstract}
Background: Pruritus Ani (PA) is the chronic itch of perianal skin and is poorly understood. There is a knowledge gap in understanding the pathophysiology and management of PA as there has been little research. The literature is sparse and of variable quality. There have been recent studies in understanding pruritic processing. We have performed a critical review of the literature concerning therapeutic inventions with the insights gained from this new understanding. In addition, an overview of PA is presented.

Method: A systematic review in accordance with Preferred Reporting Items for Systematic reviews and Meta-analysis (PRISMA) guidelines was undertaken. With the heterogeneity of the reviewed studies it was not possible to perform a true systematic review, but a critical appraisal has been performed using the same methodology.

Results: There are five randomised trials, one controlled study, one observational study, and eight case series critically appraised. The studies concerning topical and injectable intradermal steroid, topical tacrolimus and topical capsaicin have not provided evidence for their therapeutic benefit. Studies suggest methylene blue anal tattooing may beneficial in the treatment of PA.

Conclusions: The aim of this paper was a review of the best evidence available on the current treatment of PA. We set up to perform a systematic review, but were unable to due to the heterogeneity of evidence; hence a critical review was performed. There remains an evidence gap in the pathophysiology and treatment of PA. More research is needed, but there are so many unknowns about the nature of PA, this will be currently difficult to perform. The understanding of pruritus and pruritic processing is in its infancy. Newer therapies such as tacrolimus and capsaicin have failed to live up to initial promise, although they can be effective in a few. Anal tattooing shows the greatest promise.
\end{abstract}

Received date: November 14, 2016

Accepted date: January 4, 2017

Published date: January 10, 2017

Citation: Arifa Siddika. A Review of the Therapeutic Interventions in the Management of Pruritus Ani. (2017) Invest Dermatol Venerol Res 3(1): 91- 102.

DOI: $10.15436 / 2381-0858.17 .1220$

Keywords: Pruritus; Itching; Anus; Ani; Anal canal; Tattooing

\section{Introduction}

Pruritus Ani (PA) is defined as the chronic intense itch of the perianal skin and is classified as idiopathic when no apparent cause is found. There is no reliable data on the incidence of PA, but the prevalence is reported to be $1 \%-5 \%^{[1]}$. PA is four times more likely to occur in men. Studies of the age group most likely to suffer from PA have reported inconsistent results, ranging from the second to sixth decade of life ${ }^{[2]}$. Most sufferers have a relapsing and remitting course, but some have severe symptoms that have a significant impact on quality of life. PA is one of those symptoms that can be managed by a variety of clinicians, mostly general practitioners, dermatologists and colorectal surgeons. Unfortunately, like irritable bowel syndrome and chronic pain syndromes, there is an underestimation of how significantly PA can affect quality of life. In addition, due to a scientific knowledge gap, many clinicians are not empathetic towards this patient group. At worst, some clinicians consider that patients are in some way to blame for their condition, especially when available therapies have not been successful. 
PA has historically been considered a symptom rather than a diagnosis, but the understanding of pruritic processing has developed recently and may challenge this. The limited evidence base has led to multiple theories about how perianal itch was the end result of multiple seemingly unrelated triggers. This evidence base must be interpreted critically. In a recent landmark psychology paper ${ }^{[3]}$, publication bias was shown to have an even greater impact than previously thought. In this work, 100 studies were reproduced, following the methods used in the original studies as closely as possible. Ninety-five of the original studies, but only a third of the replication studies were reported to have statistically significant results. Even when the data from the original and replication studies were combined, only two thirds yielded statistically significant results. Clinical studies in other fields are probably affected by reproducibility and publication bias to a similar extent. In a field of research as small as PA, publication bias and reproducibility may be even more of a problem, and all published data should be viewed critically.

The aim of this review was to present an overview of PA and its management, to critically appraise the evidence base for therapeutic interventions used in the management of PA. We have also presented the current understanding of pruritic processing and its neuronal pathways, as these have changed considerably and have a bearing on the analysis of the available research.

\section{Methodology}

A systematic review in accordance with Preferred Reporting Items for Systematic reviews and Meta-analysis (PRISMA) guidelines was undertaken ${ }^{[4]}$. A search of indexed citations from MEDLINE, Cochrane library and PUBMED databases was undertaken using the following terms; "Pruritus Ani", "anal itching", "anal irritation", "pruritus AND (anal or anus or ani or ano)", "itch* AND (anal or anus)" in October 2015. In addition, a "related articles" search, forward citation functions was performed and the references of published articles reviewed. All abstracts were reviewed and the full texts of all potentially relevant papers were considered. Only peer-reviewed publications in the English language were included in the review. Study quality was assessed according to the recommendations of the strengthening the reporting of observational studies in epidemiology (STROBE) statement ${ }^{[5]}$. Author and year of publication, number of patients within the study, method and treatments of pruritus ani were extracted from each article included in the review.

Literature searches have found only 283 publications involving PA over a 90 -year period, with the majority being abstracts, review articles or opinions. Due to heterogeneity of the evidence identified it has not been possible to carry out a formal systematic review (Figure 1). However, the same methodology has been used to produce a critical review of literature on management of PA.

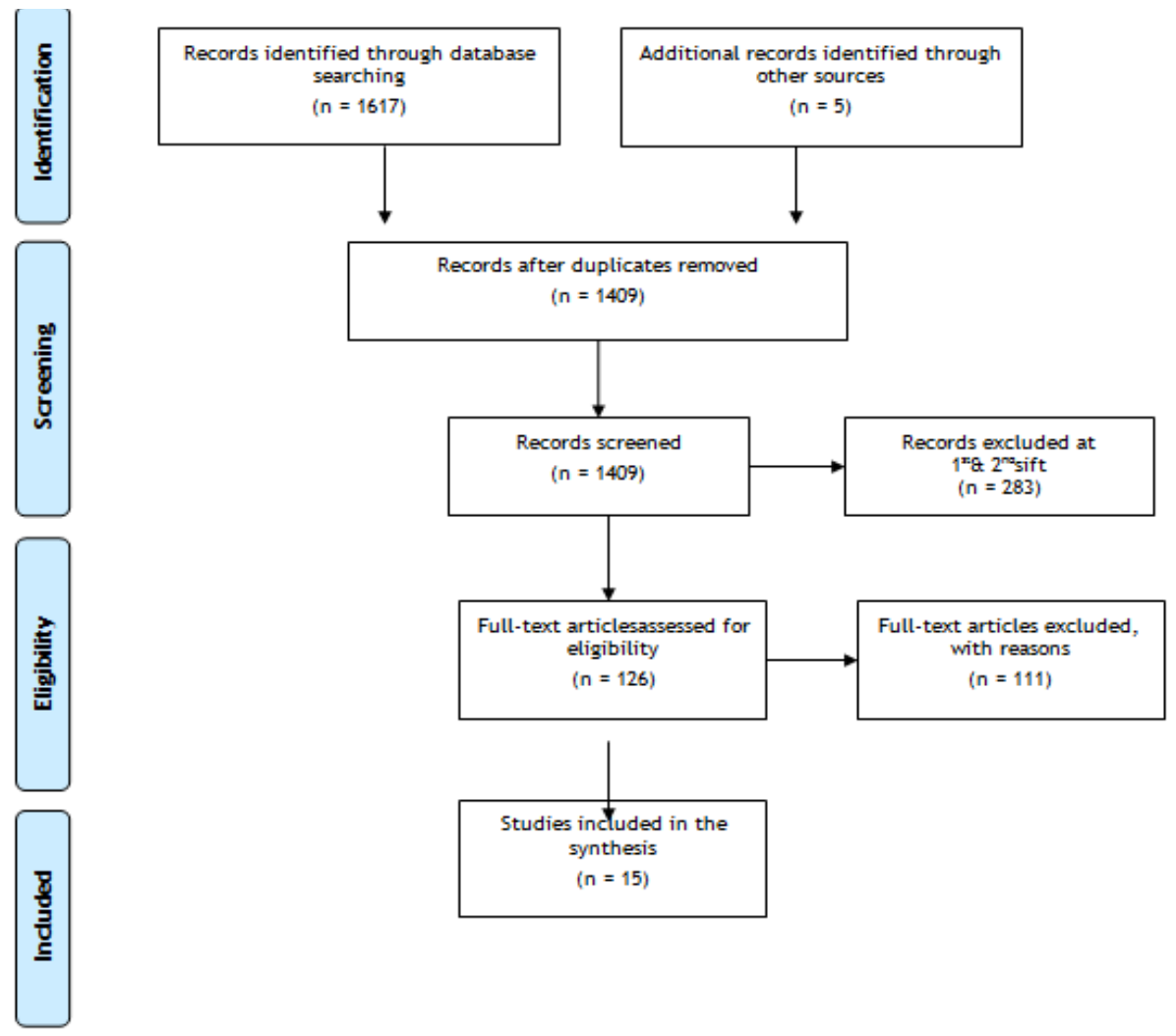

\section{General processing of pruritus}

Itch processing is highly complex, but is not fully understood. There are four subtypes of itch, i.e., pruriceptive, neurogenic, neuropathic and psychogenic ${ }^{[6]}$. Pruriceptive itch is the classical itch caused by dermal sensation and injury, chemical stimulation, dry skin, dermatological conditions, infestations, and histamine release. Neurogenic itch is caused by systemic conditions such as liver disease and renal failure where there is direct activation of higher centres of itch with no abnormality of itch processing. Neuropathic itch results from the pathological activation of itch processing in certain neurological disorders such as peripheral neuropathy, post-herpetic neuralgia, nerve compression and multiple sclerosis. Psychogenic itch may occur with some psychological conditions that involve primary 
scratching and subsequent itch.

\section{Pruriceptive mediators}

Many mediators are involved in the processing of $\operatorname{itch}^{[7,8]}$. Histamine, serine proteases, neuropeptides, neurotrophins and several inflammatory mediators have roles in histamine-dependent and histamine-independent processing of itch, with some having roles in both systems. Inflammatory mediators and neurotrophins initiate peripheral itch sensitisation in histamine-dependent and histamine-independent pruritic pathways; similar to the sensitisation they produce in nociceptive inflammatory hyperalgesia ${ }^{[7]}$.

\section{Pruriceptive receptors}

Pruriceptors (itch receptors) are subsets of sensory C-fibres and A-fibres that process both histamine-dependent and histamine-independent itch via diverse neuronal processing pathways ${ }^{[9]}$. Pain and itch are processed in the dorsal horn and have some interconnection, with pain-processing neurons actively inhibiting itch neurons. This explains why morphine reduces pain and has the side effect of itch ${ }^{[10]}$. Processing of pain and itch occurs in similar regions of the brain, but with different activation patterns. Further, the cerebral activation patterns found with histamine differ from those of other pruriceptors.

The scratch-itch-scratch cycle may arise from serotonin receptors ${ }^{[7]}$. When an itch is scratched, the ensuing pain results in release of serotonin, which reduces pain. It does this by activating the serotonin $1 \mathrm{~A}$ receptor in the spinal cord, but also stimulates a co-located receptor for the gastrin-dependent peptide that induces itch, thus completing the scratch-itch-scratch cycle. Studies of drugs targeting these receptors are underway, but the complex nature of the interactions suggests there will be no magic bullet therapy.

Several other receptors are involved in processing of pruritus, including the transient receptor potential (TRP) channels, which have a crucial role in the processing of pain and itch, but research is yet to be translated into clinical benefit. Of note some TRP channels are involved in regulation of the epidermal barrier.

Similar to nociception, there does seem to some pruritic central sensitisation in some individuals with no neurogenic equivalent. Our view of PA being purely a symptom may need to change with this knowledge.

\section{Anorectal disorders}

Twenty-five to fifty-five per cent of patients with PA have been reported to have an anorectal disorder ${ }^{[11-15]}$. These include haemorrhoids, fistulae, fissures, skin tags, warts, proctitis, neoplasia and rectal prolapse (internal or external). It is recommended to treat of all co-existing a norectal conditions ${ }^{[13,14]}$. Pironeet al. suggested that surgery for benign anorectal conditions can eliminate perianal fungal infection and both measures together reduce pruritus ${ }^{[16]}$.

\section{Anal seepage and soiling}

Any factor that increases occult or overt faecal soiling augments exposure of the perianal skin to pruritogens. Caplan's skin-patch test on the perineum and arm (using autologous faeces) produced perianal itch in one third of patients with PA and in $53 \%$ of subjects without $\mathrm{PA}^{[17]}$. Studies have also found that $41 \%-50 \%$ of patients with PA had loose stools and at least once-weekly faecal soiling ${ }^{[12,18]}$. In an anorectal physiology study of patients with PA, exaggerated recto-anal inhibitory reflexes and earlier faecal incontinence were noted. Coffee lowers anal resting pressures in over $70 \%$ of cases $^{[12]}$. Faecal seepage may also occur after anal surgery or be caused by high-grade internal rectal prolapse ${ }^{[19]}$.

\section{Perianal infections}

The importance of bacterial and fungal infection in PA is unclear. Certain infections certainly need therapy; Dermatophytes $^{[21]}$, Threadworms, Erythrasma ${ }^{[21]}$, sexually transmitted diseases $^{[22,23]}$. However, previous studies have grown multiple species of fungi and bacteria from perianal skin that were thought to be causative of $\mathrm{PA}^{[20]}$. However, current microbiological thinking suggests commensal dermal bacteria colonisation does not constitute an infection even if the species is pathogenic. The maintenance and healing of skin integrity is the critical step $^{[22,23]}$.

\section{Dermatoses and hypersensitivity}

All dermatoses can occur in the perineum and may look different from its appearance elsewhere. Psoriasis has been found in 5\%-55\% of patients with $\mathrm{PA}^{[12-14]}$. In lichen sclerosus, the skin appears white, atrophic, wrinkled and histology is diagnostic and is commonly found in the perineum. Perianal Paget's disease and Bowen's disease also have associated itch.

Chemicals found in everyday cleansing and therapeutic preparations, including creams, soaps, wet wipes, sanitary towels, toiletry sprays, deodorants and toilet paper dye, along with medications, such as topical glyceryl trinitrate, local anaesthetic preparations and haemorrhoidal medications can sensitise and cause contact dermatitis ${ }^{[13,14]}$. Sensitising chemicals have also been found in topical steroid preparations ${ }^{[23-25]}$. As up to $20 \%$ of patients may have allergens that can be avoided, patients should have skin patch testing with the European baseline series probably in those who have failed conservative measures ${ }^{[27]}$. It seems the biocides methyl chloroiso thiazolinone and methyl isothiazolinone may be a major contributor to this. They are water soluble agents found in many personal care products.

\section{Food and medication}

Although there have been no controlled trials investigating dietary modification in the treatment of PA, some researchers have suggested reduction of itch within 14 days if a certain food was avoide ${ }^{[11,18,22]}$. Some oral medications such as laxatives, colpermin, colchicine, quinidine, peppermint oil and antibioticsmay lead to $\mathrm{PA}^{[21]}$.

\section{Psychological influences}

In some cases, PA may be a manifestation of depression or psychological disturbance. Common mood disorders, such as anxiety and stress, and certain personality traits may contribute to $\mathrm{PA}^{[21]}$

\section{Examination and investigations}

A full examination should be performed with inspection of the perineum, proctoscopy and sigmoidoscopy. Fistula-in-ano, fissure-in-ano, haemorrhoids, prolapse (including internal rectal prolapse), skin tags, or warts should be identified.

Investigations include a culture swab and scraping of skin for bacteria and fungi respectively. As up to $20 \%$ of patients may have allergens that can be avoided, patients who fail con- 
servative measures should have skin patch testing with the European baseline series ${ }^{[26]}$. An examination under anesthesia of the anorectum with a circular anal dilator and perineal skin biopsy may be needed for refractory cases. The circular anal dilator is useful for determining the presence of internal or external rectal prolapse.

\section{Management}

The patient should be advised to cease using all chemicals, including creams, soaps, bubble baths and toilet paper, on their perineum and only use water for cleaning ${ }^{[1,21,26]}$. The patient should consider avoidance of caffeinated drinks and an elimination diet could be trialled. Hypoallergic detergents can be suggested for laundry purposes. Allergens discovered by skin patch testing should be avoided.

Acute pruritus ani is a marker of faecal seepage and requires immediate cleansing, especially for nocturnal itch, using water alone. The area should be dabbed dry with a towel. Aqueous cream and emollients can be used instead of soap if cleansing is required. Petroleum ointment, Sudocrem ${ }^{\circledR}$ or Cavilon ${ }^{\circledR}$ should be used as a barrier cream after washing ${ }^{[1,21]}$. Patients who sweat excessively can place cotton tissue on the perineum. There have been no randomised controlled trials investigating the use of cleansing or barrier creams in PA. However, individual case series have shown benefit. The sedating effect of some antihistamines may be useful in aiding sleep to reduce scratching, but has no effect on the itch itself ${ }^{[27]}$.

Any anorectal and dermatological condition found should be treated. There are case series showing that treatment of anal fissures, fistulae, haemorrhoids, skin tags and warts in patients with PA have resulted in improvement in symptoms in $25 \%-52 \%$ of cases and the better ones will be presented and discussed later ${ }^{[1-15]}$.

Patients with looser stools should be trialled with loperamide and fibre supplementation and those with diarrhoea-predominant IBS could be offered probiotics or a FODMAP diet ${ }^{[1,21]}$. There are no studies of the treatment of faecal incontinence in PA. A high-grade internal rectal prolapse (IRP) by prolapsing into the anal canal has recently been suggested to be a cause of occult faecal seepage ${ }^{[28]}$, but there is no case series to support treatment of IRP in the management of PA.

\section{Review of evidence}

The evidence base for treatments in PA is limited. There are five randomised trials, one controlled study, one observational study, and eight case series are summarised in Table 1 and their methodological quality is variable ${ }^{[29-42]}$. One major issue is that there is no consistent definition of idiopathic PA.

Table 1: Review of Evidence.

\begin{tabular}{|c|c|c|c|c|c|c|c|}
\hline $\begin{array}{l}\text { Study } \\
\text { author, } \\
\text { year } \\
\text { (citation) }\end{array}$ & Study type & $\begin{array}{l}\text { Clinical } \\
\text { condition/ } \\
\text { popula- } \\
\text { tion (n=) } \\
\text { Setting(s)/ } \\
\text { Provider(s) } \\
\text { Coun- } \\
\text { try(ies) }\end{array}$ & $\begin{array}{l}\text { Intervention } \\
\text { Duration of } \\
\text { treatment and } \\
\text { post-treatment } \\
\text { follow up }\end{array}$ & $\begin{array}{l}\text { Compar- } \\
\text { ator }\end{array}$ & Outcomes/measures Results & $\begin{array}{l}\text { Author conclu- } \\
\text { sions }\end{array}$ & Limitations \\
\hline $\begin{array}{l}\text { Al- } \\
\text { Ghnaniem } \\
\text { et al. } 2007\end{array}$ & $\begin{array}{l}\text { Ran- } \\
\text { domised, } \\
\text { double } \\
\text { blind pla- } \\
\text { cebo-con- } \\
\text { trolled } \\
\text { crossover } \\
\text { pilot trial }\end{array}$ & $\begin{array}{l}\text { Adults ( }>18 \\
\text { yrs) with } \\
\text { idiopathic } \\
\text { pruritus ani. } \\
\mathrm{n}=11 \text { ( } \mathrm{n}=10 \\
\text { completed) } \\
\text { Special } \\
\text { clinic } \\
\text { run by a } \\
\text { surgeon and } \\
\text { a dermatol- } \\
\text { ogist. UK }\end{array}$ & $\begin{array}{l}1 \% \text { hydrocorti- } \\
\text { sone ointment } \\
+ \text { advice on } \\
\text { hygiene } \\
\text { Twice daily for } \\
\text { two weeks. } \\
2 \text { weeks' run-in } \\
\text { period } \\
2 \text { weeks' treat- } \\
\text { ment/placebo } \\
2 \text { weeks' wash- } \\
\text { out period } \\
2 \text { weeks' treat- } \\
\text { ment/placebo }\end{array}$ & $\begin{array}{l}\text { Placebo } \\
\text { ointment } \\
+ \text { advice } \\
\text { on hy- } \\
\text { giene }\end{array}$ & $\begin{array}{l}\text { Symptoms } \\
\text { Large significant reduction ( } 68 \% \text { ) } \\
\text { in the severity of the itch with } \\
\text { hydrocortisone compared with } \\
\text { placebo (mean difference } 19.7 \\
\text { mm, } 95 \% \text { CI } 7 \text { to } 32.4, \mathrm{p}=0.019 \text { ). } \\
\text { (primary outcome; } 100 \mathrm{~mm} \text { visual } \\
\text { analogue scale (VAS)) } \\
\text { QoL: } \\
\text { Large ( } 75 \% \text { ) but non-significant } \\
\text { improvement in QoLwith hydro- } \\
\text { cortisone compared with placebo } \\
\text { (mean difference in reduction in } \\
\text { Dermatology Life Quality Index } \\
\text { score: } 1.8,95 \% \text { CI } 0.2 \text { to } 3.4 \text {, } \\
\text { p=0.065). (Secondary outcome) } \\
\text { Safety: } \\
\text { No side effects were reported } \\
\text { Other: } \\
\text { Large and significant improve- } \\
\text { ment in clinical appearance of } \\
\text { the perianal skin measured by the } \\
\text { Eczema Area and Severity Index } \\
\text { (EASI). } \\
\text { Reduction in the EASI score by } \\
\text { a median of } 2 \text {, range } 1-5, p<0.01 . \\
\text { (Secondary outcome) }\end{array}$ & $\begin{array}{l}\text { This study } \\
\text { demonstrat- } \\
\text { ed that } 1 \% \\
\text { hydrocortisone } \\
\text { ointment is an } \\
\text { effective and } \\
\text { safe treatment } \\
\text { for PA in the } \\
\text { short term. }\end{array}$ & $\begin{array}{l}\text { Small sample } \\
\text { size. } \\
\text { No power } \\
\text { calculations } \\
\text { were done } \\
\text { as this was a } \\
\text { pilot study. } \\
\text { The study } \\
\text { design did } \\
\text { not allow for } \\
\text { assessment of } \\
\text { the long term } \\
\text { effects. }\end{array}$ \\
\hline
\end{tabular}




\begin{tabular}{|c|c|c|c|c|c|c|c|}
\hline $\begin{array}{l}\text { Botterill } \\
\text { and Sagar } \\
2002\end{array}$ & $\begin{array}{l}\text { Case } \\
\text { series }\end{array}$ & $\begin{array}{l}\text { Adults ( }>18 \\
\text { yrs) with } \\
\text { chronic } \\
\text { pruritus ani } \\
\text { who failed } \\
\text { to respond } \\
\text { to standard } \\
\text { primary, } \\
\text { dermato- } \\
\text { logical and } \\
\text { colorectal } \\
\text { care. } \\
\text { n=25 (con- } \\
\text { secutive } \\
\text { patients) } \\
\text { UK }\end{array}$ & $\begin{array}{l}\text { Intradermal } \\
\text { injection } 20 \mathrm{ml} \\
\text { solution com- } \\
\text { prising } 15 \mathrm{ml} \text { of } \\
1 \% \text { lignocaine } \\
\text { hydrochloride, } \\
5 \mathrm{ml} \text { of } 1 \% \\
\text { methylene blue } \\
\text { and } 100 \mathrm{mg} \text { of } \\
\text { hydrocortisone } \\
\text { Clinic review at } \\
2 \text { and } 6 \text { weeks } \\
\text { post-treatment. } \\
\text { Annual follow } \\
\text { up by phone or } \\
\text { at clinic if pa- } \\
\text { tients relapsed. } \\
\text { Median follow } \\
\text { up } 11 \text { months } \\
\text { (2-25 months). }\end{array}$ & None & $\begin{array}{l}\text { Symptoms: } \\
16 \text { patients }(64 \%) \text { were symptom } \\
\text { free after one injection } \\
8 \text { patients were symptom free } \\
\text { after second injection ( } 88 \% \text { of total } \\
\text { number) } \\
3 \text { patients ( } 12 \%) \text { did not respond } \\
\text { to treatment (i.e. continued to expe- } \\
\text { rience symptoms) } \\
\text { QoL: } \\
\text { Not assessed } \\
\text { Safety: } \\
1 \text { patient }(4 \%) \text { had short term } \\
\text { complications (faecal seepage for } 2 \\
\text { days after injection) }\end{array}$ & $\begin{array}{l}\text { Intradermal } \\
\text { injection of } \\
\text { methylene blue } \\
\text { in combi- } \\
\text { nation with } \\
\text { lignocaine and } \\
\text { hydrocortisone } \\
\text { can provide } \\
\text { sustained relief } \\
\text { from symptoms } \\
\text { in people who } \\
\text { fail to respond } \\
\text { to standard } \\
\text { treatments. }\end{array}$ & $\begin{array}{l}\text { The lack of a } \\
\text { comparator } \\
\text { group reduces } \\
\text { ability to } \\
\text { determine to } \\
\text { what extent } \\
\text { improvements } \\
\text { may have } \\
\text { occurred } \\
\text { without treat- } \\
\text { ment. The } \\
\text { study did not } \\
\text { report clearly } \\
\text { how the out- } \\
\text { comes were } \\
\text { assessed, } \\
\text { or assess } \\
\text { severity of } \\
\text { symptoms. }\end{array}$ \\
\hline Gupta 2005 & $\begin{array}{l}\text { Case series } \\
\text { (based on } \\
\text { abstract) }\end{array}$ & $\begin{array}{l}\text { People with } \\
\text { pruritus ani } \\
\text { and chronic } \\
\text { anal fissure } \\
\text { having con- } \\
\text { comitant } \\
\text { hypertro- } \\
\text { phied anal } \\
\text { papillae } \\
\text { or fibrous } \\
\text { anal polyps } \\
\mathrm{n}=136 \\
\text { India }\end{array}$ & $\begin{array}{l}\text { Surgical proce- } \\
\text { dure involving } \\
\text { sphincterotomy } \\
\text { and destroying } \\
\text { of polyps or } \\
\text { papillae using a } \\
\text { radio frequency } \\
\text { device } \\
\text { Follow up: } 1 \\
\text { and } 18 \text { months }\end{array}$ & None & $\begin{array}{l}\text { Symptoms: } \\
\text { At one month, there was sig- } \\
\text { nificant reduction in pruritus } \\
\text { ( } \mathrm{p}=0.0003) \text {, discharge per anus } \\
(\mathrm{p}=0.0006 \text { ), crawling sensation in } \\
\text { the anus ( } \mathrm{p}=0.0004 \text { ) and that of } \\
\text { incomplete evacuation ( } \mathrm{p}=0.001 \text { ) } \\
\text { At } 18 \text { months follow up, } 9 \% \text { of } \\
\text { patients had recurrence of either } \\
\text { anal fissure or symptoms like } \\
\text { pruritus } \\
\text { QoL: } \\
\text { Not assessed } \\
\text { Safety: } \\
\text { Not assessed }\end{array}$ & $\begin{array}{l}\text { Removal of } \\
\text { hypertrophied } \\
\text { anal papillae } \\
\text { and fibrous } \\
\text { polyps should } \\
\text { be carried on } \\
\text { a routine basis } \\
\text { during surgical } \\
\text { treatment of } \\
\text { anal fissure. } \\
\text { This would add } \\
\text { to the effective- } \\
\text { ness and com- } \\
\text { pleteness of the } \\
\text { procedure. The } \\
\text { lack of a com- } \\
\text { parator group } \\
\text { reduces ability } \\
\text { to determine } \\
\text { to what extent } \\
\text { improvements } \\
\text { may have oc- } \\
\text { curred without } \\
\text { treatment. }\end{array}$ & $\begin{array}{l}\text { Pruritus was } \\
\text { not main fo- } \\
\text { cus of study. }\end{array}$ \\
\hline $\begin{array}{l}\text { Jensen } \\
1988\end{array}$ & $\begin{array}{l}\text { Ran- } \\
\text { domised } \\
\text { controlled } \\
\text { trial }\end{array}$ & $\begin{array}{l}\text { Patients } \\
\text { with chron- } \\
\text { ic pruritus } \\
\text { ani and } \\
\text { hypertro- } \\
\text { phied anal } \\
\text { papillae. } \\
\text { n=41. } \\
\text { Denmark }\end{array}$ & $\begin{array}{l}\text { Excision of } \\
\text { hypertrophied } \\
\text { anal papillae } \\
\text { under local an- } \\
\text { aesthesia } n=21 \\
\text { Follow up at } \\
1 \text { and } 4 \text { weeks } \\
\text { after rando- } \\
\text { misation and } \\
\text { treatment, and } \\
\text { at } 1 \text { year }\end{array}$ & $\begin{array}{l}\text { Expect- } \\
\text { ant man- } \\
\text { agement } \\
n=20\end{array}$ & $\begin{array}{l}\text { Symptoms: } \\
\text { Proctological evaluation of symp- } \\
\text { toms pre- and post- surgery } \\
\text { No significant effect was found. } \\
14(67 \%) \text { of the } 21 \text { patients in the } \\
\text { excision group were symptom free } \\
\text { at } 1 \text { year compared to } 11(55 \%) \\
\text { of the } 20 \text { patients in the expectant } \\
\text { management group ( }>0.05) \text {. } \\
\text { QoL: } \\
\text { Not assessed } \\
\text { Safety: } \\
8(42 \%) \text { of the patients in the } \\
\text { excision group developed substan- } \\
\text { tial pain within } 1 \text { week of treatment } \\
\text { compared to zero of the } 20 \text { patients } \\
\text { in the expectant management group } \\
(p<0.01)\end{array}$ & $\begin{array}{l}\text { Excision of } \\
\text { hypertrophied } \\
\text { anal papillae } \\
\text { in people with } \\
\text { chronic pruritus } \\
\text { ani showed } \\
\text { no effect at } 1 \\
\text { year compared } \\
\text { with effective } \\
\text { management, } \\
\text { and may be } \\
\text { considered } \\
\text { harmful due to } \\
\text { the high rate of } \\
\text { postoperative } \\
\text { complications. }\end{array}$ & $\begin{array}{l}\text { This report } \\
\text { did not } \\
\text { include any } \\
\text { details about } \\
\text { the method } \\
\text { of randomi- } \\
\text { sation used } \\
\text { or whether } \\
\text { there were } \\
\text { any efforts for } \\
\text { concealment } \\
\text { of allocation. } \\
\text { It is also not } \\
\text { clear whether } \\
\text { this study } \\
\text { was powered } \\
\text { to detect } \\
\text { significant } \\
\text { differences } \\
\text { between the } \\
\text { groups. }\end{array}$ \\
\hline
\end{tabular}




\begin{tabular}{|c|c|c|c|c|c|c|c|}
\hline $\begin{array}{l}\text { Kang et al. } \\
2009\end{array}$ & $\begin{array}{l}\text { Case } \\
\text { series } \\
\text { (Con- } \\
\text { ference } \\
\text { abstract) }\end{array}$ & $\begin{array}{l}\text { People with } \\
\text { chronic } \\
\text { intractable } \\
\text { pruritus ani } \\
n=100\end{array}$ & $\begin{array}{l}\text { Perianal } \\
\text { intracutaneous } \\
\text { injection of } \\
\text { Triamcinolone } \\
\text { Follow up: } 2 \\
\text { years (95\% of } \\
\text { patients were } \\
\text { assessed at } 2 \\
\text { years) }\end{array}$ & None & $\begin{array}{l}\text { Symptoms: } \\
\text { Outcomes and outcome measures } \\
\text { not reported } \\
\text { Results not reported in detail } \\
\text { "we observed full relief of itching } \\
\text { sensations" } \\
\text { QoL: } \\
\text { Not assessed } \\
\text { Safety: } \\
\text { Not reported }\end{array}$ & $\begin{array}{l}\text { Local injection } \\
\text { of Triamcino- } \\
\text { lone is simple } \\
\text { but very effec- } \\
\text { tive method to } \\
\text { treat chronic } \\
\text { intractable } \\
\text { pruritus ani. }\end{array}$ & $\begin{array}{l}\text { The lack of a } \\
\text { comparator } \\
\text { group reduces } \\
\text { ability to } \\
\text { determine to } \\
\text { what extent } \\
\text { improvements } \\
\text { may have } \\
\text { occurred } \\
\text { without treat- } \\
\text { ment. } \\
\text { Lack of data } \\
\text { for outcomes } \\
\text { and results in } \\
\text { conference } \\
\text { abstract. }\end{array}$ \\
\hline $\begin{array}{l}\text { Lysy et al. } \\
2003\end{array}$ & $\begin{array}{l}\text { Ran- } \\
\text { domised } \\
\text { place- } \\
\text { bo-con- } \\
\text { trolled } \\
\text { crossover } \\
\text { trial }\end{array}$ & $\begin{array}{l}\text { People with } \\
\text { chronic } \\
\text { idiopathic } \\
\text { intractable } \\
\text { pruritus ani } \\
\mathrm{n}=49 \\
\text { Israel }\end{array}$ & $\begin{array}{l}\text { Capsaicin } \\
\text { cream } 0.006 \% \\
\text { three times a } \\
\text { day } \mathrm{n}=22 \\
2 \text { treatment } \\
\text { phases of } 4 \\
\text { weeks separat- } \\
\text { ed with } 1 \text { week } \\
\text { washout phase } \\
\text { Follow up: } \\
\text { post treatment } \\
\text { evaluation at } 4 \\
\text { and } 9 \text { weeks } \\
\text { After } 9 \text { weeks } \\
\text { respondents } \\
\text { continued in } \\
\text { an open label } \\
\text { study with a } \\
\text { follow up every } \\
2-3 \text { months } \\
\text { (mean } 10.9 \text { (SD } \\
5.8 \text { ) months) }\end{array}$ & $\begin{array}{l}\text { Placebo } \\
\text { (menthol } \\
1 \% \text { ) } \\
\text { cream } \\
n=22\end{array}$ & $\begin{array}{l}\text { Symptoms: } \\
\text { Pre- and post-treatment clinical } \\
\text { evaluation and } 28 \text {-day patient } \\
\text { symptom diaries for both } 4 \text { weeks' } \\
\text { treatment phases } \\
\text { There was a significant effect } \\
\text { for capsaicin treatment with } 31 \text { of } \\
44 \text { patients being symptom free } \\
\text { (p<0.0001). } \\
\text { QoL: } \\
\text { Not assessed } \\
\text { Safety: } \\
\text { - Burning (side effect) score and } \\
\text { duration were significantly higher } \\
\text { following capsaicin treatment } \\
\text { (p<0.001) than with placebo } \\
\text { - } 4 \text { people dropped out due to } \\
\text { intolerable burning side effects, and } \\
\text { one due to urticaria }\end{array}$ & $\begin{array}{l}\text { Perianal } \\
\text { application of } \\
0.006 \% \text { capsa- } \\
\text { icin cream is a } \\
\text { safe and effec- } \\
\text { tive treatment } \\
\text { of chronic idio- } \\
\text { pathic intracta- } \\
\text { ble pruritus ani. }\end{array}$ & $\begin{array}{l}\text { The report did } \\
\text { not provide } \\
\text { any details } \\
\text { about the } \\
\text { concealment } \\
\text { of allocation. } \\
\text { This study } \\
\text { randomised } \\
49 \text { patients } \\
\text { of which } 5 \\
\text { dropped in } \\
\text { the first week } \\
\text { of treatment } \\
\text { due to side } \\
\text { effects. The } \\
\text { analysis does } \\
\text { not seem to } \\
\text { account for } \\
\text { the drop outs. }\end{array}$ \\
\hline $\begin{array}{l}\text { Mentes et } \\
\text { al. } 2003\end{array}$ & $\begin{array}{l}\text { Case } \\
\text { series }\end{array}$ & $\begin{array}{l}\text { Adults with } \\
\text { intractable } \\
\text { idiopathic } \\
\text { pruriti- } \\
\text { sani. } n=30 \\
\text { Turkey }\end{array}$ & $\begin{array}{l}15 \text { ml solu- } \\
\text { tion of equal } \\
\text { volume of } 2 \% \\
\text { methylene } \\
\text { blue and } 0.5 \% \\
\text { lidocaine in- } \\
\text { jected intracu- } \\
\text { taneously and } \\
\text { subcutaneously } \\
\text { in the affected } \\
\text { perianal area. } \\
\text { Second } \\
\text { injection a } \\
\text { month later for } \\
\text { patients (n=5) } \\
\text { with partial } \\
\text { response. } \\
\text { Follow up at } \\
24 \text { hours for } \\
\text { early compli- } \\
\text { cations, at } 1 \\
\text { and } 6 \text { months, } \\
\text { and then on an } \\
\text { annual basis. }\end{array}$ & None & $\begin{array}{l}\text { Symptoms: } \\
\text { - At } 1 \text { month, } 24(80 \%) \text { were } \\
\text { symptom free, } 5 \text { had partial remis- } \\
\text { sion, and } 1 \text { patient had no change. } \\
\text { With the second injection } 28 \\
(93.3 \%) \text { were symptom free. } \\
\text { - At } 6 \text { months, } 25(83.3 \%) \text { were } \\
\text { symptom free. } \\
\text { - At } 1 \text { year, } 23(76.6 \%) \text { were } \\
\text { symptom free. } \\
\text { QoL: } \\
\text { - Not assessed } \\
\text { Safety: } \\
\text { - No major complications were } \\
\text { reported } \\
\text { Other } \\
\text { - At } 1 \text { month, symptomatic healing } \\
\text { was confirmed by observation of } \\
\text { the disappearance of perianal exco- } \\
\text { riation and lichenification. }\end{array}$ & $\begin{array}{l}\text { This study } \\
\text { showed that } \\
\text { intradermal } \\
\text { methylene blue } \\
\text { injection is a } \\
\text { safe, simple, } \\
\text { fast and efficient } \\
\text { method of treat- } \\
\text { ing intractable } \\
\text { pruritus ani. }\end{array}$ & $\begin{array}{l}\text { The lack of a } \\
\text { comparator } \\
\text { group reduces } \\
\text { ability to } \\
\text { determine to } \\
\text { what extent } \\
\text { improvements } \\
\text { may have } \\
\text { occurred } \\
\text { without treat- } \\
\text { ment. } \\
\text { Inconsistent } \\
\text { reporting of } \\
\text { the quantity } \\
\text { of methylene } \\
\text { blue through- } \\
\text { out }\end{array}$ \\
\hline
\end{tabular}




\begin{tabular}{|c|c|c|c|c|c|c|c|}
\hline $\begin{array}{l}\text { Miravalle } \\
\text { et al. } 2013\end{array}$ & $\begin{array}{l}\text { Case series } \\
\text { (con- } \\
\text { ference } \\
\text { abstract) }\end{array}$ & $\begin{array}{l}\text { Adults }>18 \\
\text { yrs with } \\
\text { idiopathic } \\
\text { pruritus ani } \\
\mathrm{n}=24 \\
\text { Colorectal } \\
\text { surgery } \\
\text { centre } \\
\text { Argentina }\end{array}$ & $\begin{array}{l}\text { Therapeutic } \\
\text { algorithm: } \\
\text { Step 1. Hygiene } \\
\text { and lifestyle } \\
\text { modification } \\
\text { Step 2. Topical } \\
\text { hydrocortisone } \\
\text { Step. } 3 \text { Topical } \\
\text { capsaicin } \\
\text { Follow up: } 6 \\
\text { months }\end{array}$ & None & $\begin{array}{l}\text { Symptoms } \\
\text { - Pruritus was assessed by } 100 \mathrm{~mm} \\
\text { Visual Analogue Scale (VAS) } \\
\text { - At } 6 \text { months, } 22 \text { (91.7\%; } 95 \% \\
\text { CI } 73-99) \text { were symptom free. } 2 \\
\text { patients ( } 8.3 \% \text {; } 95 \% \text { CI 1-27) had } \\
\text { partial response. } \\
\text { QoL } \\
\text { - Not assessed } \\
\text { Safety } \\
\text { - Not assessed }\end{array}$ & $\begin{array}{l}\text { The treatment } \\
\text { response was } \\
\text { favourable in all } \\
\text { patients. Symp- } \\
\text { toms remission } \\
\text { was consistent } \\
\text { with perianal } \\
\text { skin recovery. }\end{array}$ & $\begin{array}{l}\text { The lack of a } \\
\text { comparator } \\
\text { group reduces } \\
\text { ability to } \\
\text { determine to } \\
\text { what extent } \\
\text { improvements } \\
\text { may have } \\
\text { occurred } \\
\text { without treat- } \\
\text { ment. } \\
\text { This was a } \\
\text { conference } \\
\text { abstract so the } \\
\text { therapeutic } \\
\text { algorithm was } \\
\text { not described } \\
\text { in detail. }\end{array}$ \\
\hline $\begin{array}{l}\text { Novak et } \\
\text { al. } 1976\end{array}$ & $\begin{array}{l}\text { Controlled } \\
\text { clinical } \\
\text { trial }\end{array}$ & $\begin{array}{l}\text { Patients } \\
\text { with } \\
\text { chemically } \\
\text { induced } \\
\text { pruritusani } \\
\mathrm{N}=\text { not } \\
\text { reported in } \\
\text { abstract }\end{array}$ & $\begin{array}{l}\text { Single intra- } \\
\text { venous doses } \\
\text { of } 100,200, \\
\text { or } 400 \mathrm{mg} \text { of } \\
\text { hydrocorti- } \\
\text { sone sodium } \\
\text { succinate and } \\
\text { hydrocortisone } \\
\text { sodium phos- } \\
\text { phate }\end{array}$ & Placebo & $\begin{array}{l}\text { Aetiology } \\
\text { - Chemically induced pruritus ani } \\
\text { - More subjects receiving hy- } \\
\text { drocortisonesodium phosphate } \\
\text { experienced systemic or localized } \\
\text { adverse effects than those receiving } \\
\text { hydrocortisonesodiumsuccinate } \\
\text { - } 16 \text { of } 18 \text { subjects medicated with } \\
\text { hydrocortisone sodium phosphate } \\
\text { experienced side effects of burning } \\
\text { and itching } \\
\text { - } 1 \text { subject of } 6 \text { treated with place- } \\
\text { bo (saline) and none in the sodium } \\
\text { succinate group. }\end{array}$ & $\begin{array}{l}\text { The side effect } \\
\text { of burning and } \\
\text { itching in the } \\
\text { anorectal area } \\
\text { is attributed to } \\
\text { the phosphate } \\
\text { steroid and } \\
\text { appears to last } \\
\text { as long as it } \\
\text { takes to convert } \\
\text { to cortisol. }\end{array}$ & $\begin{array}{l}\text { The abstract } \\
\text { does not dis- } \\
\text { cuss any other } \\
\text { factors that } \\
\text { could have } \\
\text { contributed to } \\
\text { occurrence of } \\
\text { pruritus ani. } \\
\text { Limited data } \\
\text { on measures } \\
\text { used. }\end{array}$ \\
\hline $\begin{array}{l}\text { Oztaz et al. } \\
2003\end{array}$ & $\begin{array}{l}\text { Prospec- } \\
\text { tive obser- } \\
\text { vational } \\
\text { study }\end{array}$ & $\begin{array}{l}\text { People with } \\
\text { idiopathic } \\
\text { pruritus ani } \\
\mathrm{n}=60 \\
\text { University } \\
\text { hospital de- } \\
\text { partment of } \\
\text { dermatolo- } \\
\text { gy Turkey }\end{array}$ & $\begin{array}{l}\text { Topical steroids } \\
\text { (Advantan } \\
\text { cream) twice } \\
\text { daily for two } \\
\text { weeks } n=28 \\
\text { Follow up : two } \\
\text { weeks }\end{array}$ & $\begin{array}{l}\text { Liquid } \\
\text { cleanser } \\
\text { (Protex) } \\
\text { twice } \\
\text { daily } \\
\text { for two } \\
\text { weeks } \\
n=32\end{array}$ & $\begin{array}{l}\text { Symptoms } \\
\text { - Patient reported pruritus scores } \\
(0-10,0 \text { - no pruritus, } 10 \text { most } \\
\text { severe pruritus }) \\
\text { - At } 2 \text { weeks treatment was } \\
\text { effective in } 26(92.3 \%) \text { patients } \\
\text { in the topical steroid group and in } \\
29(90.6 \%) \text { in the liquid cleanser } \\
\text { group. There was no statistically } \\
\text { significant difference between the } \\
\text { two groups, p }>0.05 \text {. } \\
\text { QoL } \\
\text { - Not assessed } \\
\text { Safety } \\
\text { - No side effects were detected } \\
\text { in both groups at the end of the } \\
\text { treatment }\end{array}$ & $\begin{array}{l}\text { Perianal cleans- } \\
\text { ing is as effec- } \\
\text { tive as topical } \\
\text { corticosteroids } \\
\text { in the treatment } \\
\text { of idiopathic } \\
\text { pruritus ani. } \\
\text { Mild cleansers } \\
\text { could be used } \\
\text { as a safe first } \\
\text { step treatment } \\
\text { for controlling } \\
\text { perianal itching. }\end{array}$ & $\begin{array}{l}\text { This was a } \\
\text { prospective } \\
\text { observational } \\
\text { study with a } \\
\text { potential for } \\
\text { allocation } \\
\text { bias. The } \\
\text { results were } \\
\text { not reported } \\
\text { in detail. The } \\
\text { outcomes } \\
\text { were mea- } \\
\text { sured subjec- } \\
\text { tively. The } \\
\text { patients were } \\
\text { not followed } \\
\text { up after the } \\
\text { post treatment } \\
\text { evaluation at } \\
2 \text { weeks. }\end{array}$ \\
\hline
\end{tabular}




\begin{tabular}{|c|c|c|c|c|c|c|c|}
\hline $\begin{array}{l}\text { Samalavi- } \\
\text { cius et al. } \\
2012\end{array}$ & $\begin{array}{l}\text { Case } \\
\text { series }\end{array}$ & $\begin{array}{l}\text { People with } \\
\text { intractable } \\
\text { idiopathic } \\
\text { pruritus ani } \\
\text { n=10 } \\
\text { University } \\
\text { hospital } \\
\text { Lithuania }\end{array}$ & $\begin{array}{l}15 \mathrm{ml} 1 \% \\
\text { methylene blue } \\
\text { solution ( } 10 \mathrm{ml} \\
2 \% \text { methylene } \\
\text { blue, } 5 \mathrm{ml} \text { sa- } \\
\text { line, } 5 \mathrm{ml} 2 \% \\
\text { lidocaine solu- } \\
\text { tion) injected } \\
\text { intradermally } \\
\text { in the perianal } \\
\text { itching area } \\
\text { Follow up at } 4 \\
\text { weeks (clinical } \\
\text { examination) } \\
\text { and by tele- } \\
\text { phone inter- } \\
\text { view at } 6 \text { and } \\
12 \text { months, and } \\
\text { then on a yearly } \\
\text { basis. }\end{array}$ & None & $\begin{array}{l}\text { Symptoms } \\
\text { - Patient symptom score ( } 1 \text { - } 5,1= \\
\text { worst) was used to measure itching } \\
\text { severity } \\
\text { - All } 10 \text { patients were symptom } \\
\text { free at } 4 \text { weeks after treatment } \\
\text { - At long term follow up, median } \\
47 \text { months (range } 29-60) 8 \text { patients } \\
\text { reported recurrence of symptoms. } \\
\text { QoL } \\
\text { - Not assessed } \\
\text { Safety } \\
\text { - No severe side effects were } \\
\text { reported. Mild side effects related } \\
\text { to sensory cutaneous innervation } \\
\text { were reported in all patients within } \\
\text { the first } 4 \text { weeks. }\end{array}$ & $\begin{array}{l}\text { Intradermal } \\
\text { injection of } \\
1 \% \text { methylene } \\
\text { blue solution is } \\
\text { associated with } \\
\text { a positive effect } \\
\text { om idiopathic } \\
\text { pruritus ani in } \\
\text { the short term } \\
\text { ( } 4 \text { weeks). Long } \\
\text { term success } \\
\text { rate was } 20 \% \text {. }\end{array}$ & $\begin{array}{l}\text { The lack of a } \\
\text { comparator } \\
\text { group reduces } \\
\text { ability to } \\
\text { determine to } \\
\text { what extent } \\
\text { improvements } \\
\text { may have } \\
\text { occurred } \\
\text { without } \\
\text { treatment. A } \\
\text { patient-report- } \\
\text { ed symptom } \\
\text { score (1 - 5, } \\
1=\text { worst) } \\
\text { was used to } \\
\text { measure the } \\
\text { outcome. No } \\
\text { statistical } \\
\text { comparisons } \\
\text { were carried } \\
\text { out. }\end{array}$ \\
\hline $\begin{array}{l}\text { Sutherland } \\
\text { et al. } 2008\end{array}$ & $\begin{array}{l}\text { Case } \\
\text { series }\end{array}$ & $\begin{array}{l}\text { Adults with } \\
\text { refractory } \\
\text { idiopathic } \\
\text { pruritus ani } \\
\text { n=49 } \\
\text { New Zea- } \\
\text { land }\end{array}$ & $\begin{array}{l}\text { Solution of } \\
10 \mathrm{ml} 1 \% \\
\text { methylene } \\
\text { blue, } 20 \mathrm{ml} \text { of } \\
0.5 \% \text { marcain } \\
\text { with } 1: 200000 \\
\text { adrenaline and } \\
1 \mathrm{ml} \text { methyl- } \\
\text { prednisolone } \\
1 \text { intradermal } \\
\text { injection } \\
\text { Review at } 4 \\
\text { weeks } \\
\text { Follow up at } 8 \\
\text { weeks } \\
\text { (waiting for } \\
\text { long term } \\
\text { follow up) }\end{array}$ & None & $\begin{array}{l}\text { Symptoms } \\
\text { - Patient symptom score }(1-5,1= \\
\text { worst) was used to measure itching } \\
\text { severity } \\
\text { - } 30(57 \%) \text { patients were symptom } \\
\text { free after one treatment; } 4 \text { of the } \\
\text { rest had a second injection and } \\
\text { were symptom free (a total of } 65 \%) \\
\text { - } 96 \% \text { reported improvement } \\
\text { QoL } \\
\text { - Not assessed } \\
\text { Safety } \\
\text { - } 7 \text { (14\%) patients had short } \\
\text { term changes in continence and } 2 \\
\text { experienced decrease in perianal } \\
\text { sensation. }\end{array}$ & $\begin{array}{l}\text { Intradermal } \\
\text { methylene } \\
\text { blue injection } \\
\text { is effective for } \\
\text { treatment of } \\
\text { refractory idio- } \\
\text { pathic pruritus } \\
\text { ani. }\end{array}$ & $\begin{array}{l}\text { The lack of a } \\
\text { comparator } \\
\text { group reduces } \\
\text { ability to } \\
\text { determine to } \\
\text { what extent } \\
\text { improvements } \\
\text { may have } \\
\text { occurred } \\
\text { without treat- } \\
\text { ment. } \\
\text { A patient-re- } \\
\text { ported symp- } \\
\text { tom score (1 } \\
\text { - 5, } 1=\text { worst) } \\
\text { was used to } \\
\text { measure the } \\
\text { outcome. No } \\
\text { statistical } \\
\text { comparisons } \\
\text { were carried } \\
\text { out. }\end{array}$ \\
\hline Suys 2012 & $\begin{array}{l}\text { Ran- } \\
\text { domised } \\
\text { dou- } \\
\text { ble-blind } \\
\text { place- } \\
\text { bo-con- } \\
\text { trolled } \\
\text { crossover } \\
\text { trial (Let- } \\
\text { ter) }\end{array}$ & $\begin{array}{l}\text { People with } \\
\text { resistant } \\
\text { idiopathic } \\
\text { pruritus ani } \\
\mathrm{n}=21 \\
\text { Belgium }\end{array}$ & $\begin{array}{l}0.1 \% \text { Tacroli- } \\
\text { mus ointment } \\
\text { once daily } \\
n=10 \\
4 \text { weeks' treat- } \\
\text { ment/placebo } \\
1 \text { week wash- } \\
\text { out period } \\
4 \text { weeks' place- } \\
\text { bo/treatment } \\
\text { No long-term } \\
\text { follow up }\end{array}$ & $\begin{array}{l}\text { Placebo } \\
\text { (petro- } \\
\text { latum) } \\
\text { once } \\
\text { daily } \\
n=11\end{array}$ & $\begin{array}{l}\text { Symptoms } \\
\text { - Intensity and frequency of anal } \\
\text { itch } \\
\text { - Significant positive effect of } \\
\text { tacrolimus vs placebo on both } \\
\text { itch intensity ( }-1.73 \text { vs }-0.005 \text {, } \\
\text { p=0.044) and frequency (-1.71 vs } \\
0.03, p=0.019) \\
\text { QoL } \\
\text { - Dermatology Life Quality Index } \\
\text { results were positive but not sig- } \\
\text { nificant for tacrolimus vs placebo } \\
\text { (-3.77 vs }-1.04, p=0.146) \\
\text { Safety } \\
\text { - No side effects were reported }\end{array}$ & $\begin{array}{l}\text { Tacrolimus } \\
0.1 \% \text { ointment } \\
\text { may be effective } \\
\text { treatment for } \\
\text { idiopathic } \\
\text { pruritus ani with } \\
68 \% \text { of patients } \\
\text { improving after } \\
2 \text { weeks. }\end{array}$ & $\begin{array}{l}\text { The study } \\
\text { design did } \\
\text { not allow for } \\
\text { assessment of } \\
\text { the long term } \\
\text { effects. } \\
\text { This study } \\
\text { was published } \\
\text { as a letter so } \\
\text { the report was } \\
\text { very brief. }\end{array}$ \\
\hline
\end{tabular}




\begin{tabular}{|c|c|c|c|c|c|c|c|}
\hline $\begin{array}{l}\text { Ucak et al. } \\
2013\end{array}$ & $\begin{array}{l}\text { Ran- } \\
\text { domised } \\
\text { place- } \\
\text { bo-con- } \\
\text { trolled } \\
\text { crossover } \\
\text { trial }\end{array}$ & $\begin{array}{l}\text { Adults }>18 \\
\text { yrs with } \\
\text { persistent } \\
\text { pruritus ani } \\
\text { and atopic } \\
\text { dermatitis } \\
\mathrm{n}=32 \text { Tur- } \\
\text { key }\end{array}$ & $\begin{array}{l}0.03 \% \text { tacroli- } \\
\text { mus ointment } \\
\text { twice daily } \\
n=16 \\
4 \text { weeks' treat- } \\
\text { ment/placebo } \\
2 \text { week wash- } \\
\text { out period } \\
4 \text { weeks' place- } \\
\text { bo/treatment } \\
\text { Follow up: } 8 \\
\text { weeks after } \\
\text { treatment com- } \\
\text { pletion }\end{array}$ & $\begin{array}{l}\text { Placebo } \\
\text { (Vase- } \\
\text { line) } \\
\text { twice } \\
\text { daily } \\
\mathrm{n}=16\end{array}$ & $\begin{array}{l}\text { Symptoms } \\
\text { - Patient perianal itching score ( } 0 \\
\text { to } 3,0=\text { none to } 3=\text { severe); primary } \\
\text { outcome } \\
\text { - There was a statistically signif- } \\
\text { icant decrease in the itching score } \\
\text { for the tacrolimus group compared } \\
\text { to the placebo group at weeks } 4 \text { and } \\
6 \text { ( } \mathrm{p}=0.001 \text { ). } \\
\text { - At } 18 \text { weeks follow up the } \\
\text { relapse rates were high in both } \\
\text { groups } 13 \text { ( } 81 \% \text { ) in the tacrolimus } \\
\text { group and } 11 \text { ( } 69 \% \text { ) in the placebo } \\
\text { group. } \\
\text { QoL } \\
\text { - The Dermatology Life Quality } \\
\text { Index score showed significant im- } \\
\text { provement at weeks } 4 \text { and } 6 \text { ( } p=001 \\
\text { and } p=0.008 \text { respectively). } \\
\text { Safety } \\
\bullet \text { No significant side effects were } \\
\text { identified } \\
\text { Other } \\
\text { Eczema Area and Severity Index } \\
\text { (EASI)showed significant improve- } \\
\text { ment at weeks } 4 \text { and } 6 \text { ( } p=0.001 \\
\text { and } p=0.002 \text { respectively) }\end{array}$ & & \\
\hline $\begin{array}{l}\text { Xie and } \\
\text { Lan } 2014\end{array}$ & $\begin{array}{l}\text { Case series } \\
\text { (Con- } \\
\text { ference } \\
\text { abstract) }\end{array}$ & $\begin{array}{l}\text { Adult male } \\
\text { patients } \\
\text { with } \\
\text { pruritus ani } \\
\mathrm{N}=41 \\
\text { University } \\
\text { hospital } \\
\text { China }\end{array}$ & $\begin{array}{l}0.1 \% \text { tacrolim- } \\
\text { us ointment } \\
\text { Duration of } \\
\text { treatment: not } \\
\text { reported } \\
\text { Follow up: not } \\
\text { reported }\end{array}$ & None & $\begin{array}{l}\text { Symptoms } \\
\text { - Severity of pruritus (SP) score } \\
\text { was found to be reduced signifi- } \\
\text { cantly in } 41 \text { patients (no data were } \\
\text { provided in the abstract) } \\
\text { QoL } \\
\text { - Not assessed } \\
\text { Safety } \\
34(82.9 \%) \text { showed capsaicin-like } \\
\text { response (i.e burning with conse- } \\
\text { quent rapid amelioration of pruritus } \\
\text { or burning sensation) }\end{array}$ & $\begin{array}{l}\text { Topical tac- } \\
\text { rolimus may } \\
\text { rapidly inhibit } \\
\text { or alleviate itch } \\
\text { in patients with } \\
\text { pruritus ani. }\end{array}$ & $\begin{array}{l}\text { This study } \\
\text { was a confer- } \\
\text { ence abstract } \\
\text { that didn't } \\
\text { include much } \\
\text { detail about } \\
\text { the popula- } \\
\text { tion, interven- } \\
\text { tion, outcome } \\
\text { measures and } \\
\text { follow up. } \\
\text { The lack of } \\
\text { a comparator } \\
\text { group reduces } \\
\text { ability to } \\
\text { determine to } \\
\text { what extent } \\
\text { improvements } \\
\text { may have } \\
\text { occurred } \\
\text { without treat- } \\
\text { ment. }\end{array}$ \\
\hline
\end{tabular}

Dermatologists or colorectal surgeons have conducted clinical studies on PA and their respective views of the anorectal anatomy and physiology are very different. The striking feature of the studies conducted by these two groups of clinicians is the seemingly different populations of patients with distinct aetiology. Clearly, patients with obvious dermatoses would be referred to a dermatologist and those with proctological issues to a colorectal surgeon. However, subpopulations of patients referred without an obvious underlying cause will be directed according to the referrer's normal clinical practice. This suggests that the populations would continue to have an overlap, but the treatment algorithms used by dermatologists and colorectal surgeons suggest otherwise. Either there are two distinct groups of patients seen in dermatology and coloproctology or widely alternative therapies are being used to tap into anti-pruritic pathways.

\section{Topical and intradermal steroids}

Al-Ghnaniem et al., compared hydrocortisone $1 \%$ ointment and advice with placebo and advice in a randomised crossover trial, which reported a significant (68\%) reduction in severity of itching with hydrocortisone when compared with placebo ${ }^{[29]}$. However, the sample size was 11 and outcomes were measured at the end of 2-week treatment period with no long term follow up. In another prospective observational study, Öztaş et al., showed no difference in symptom control between topical steroids and a liquid cleanser, but both were said to be effective in $90 \%$ of cas$\mathrm{es}^{[43]}$. Although this was a larger study with 28 patients treated with topical steroids alone and 32 patients with liquid cleanser alone, this was not randomised. Furthermore, it is unclear how treatment options were selected for individual patients. There is one case series with longer term follow up. Kang et al injected 
triamcinolone intracutaneously in 100 patients and found $95 \%$ to have symptom relief after 2 years ${ }^{[38]}$. However, this was a conference abstract with no details of how symptom improvement was measured and this abstract was never followed up with a paper publication.

\section{Topical tacrolimus}

There are two randomised trials and one case series reporting the therapeutic effect of topical tacrolimus in $\mathrm{PA}^{[32-34]}$. Suys et al., performed a randomised double-blinded trial involving 22 patients with a statistically significant improvement in symptom control after 4 weeks treatment with topical tacrolim$\mathrm{us}^{[32]}$. This was published as a letter, with no long-term follow-up and provided no details of how outcomes were measured. Ucak et al., published a conference abstract describing a randomised crossover study of topical tacrolimus in 16 patients $^{[33]}$. The methodology was not described, but symptoms were assessed at the end of each 4-week intervention period and a further assessment 8 weeks after. They reported a statistically significant improvement in the topical tacrolimus intervention group as opposed to placebo intervention group, but actual numbers were not provided. In addition, the relapse rate of those that had symptomatic improvement at the 8 week review was $75 \%$. The case series by Xei et al., was an investigation into the mechanism of action of topical tacrolimus in $\mathrm{PA}^{[34]}$. This conference abstract suggested a significant symptom improvement in their 41 patients, but the period of follow up was not mentioned. Although these studies reported an improvement in symptoms, the relapse rate was high and follow-up was very short. Tacrolimus is known to produce burning itch as a side effect by indirectly activating a transient receptor potential channel namely TRPV1. The therapeutic effect of tacrolimus is therefore not by inhibition of itch, but by counter-irritation. Although the evidence to support the use of this agent is limited, the risks are minor, so it can be safely trialled.

\section{Topical capsaicin}

Topical capsaicin cream has been used for the treatment of PA, but there has only been one study of reasonable quality. Lysy et al., performed a randomised crossover study, which showed significant improvement in PA symptoms ${ }^{[31]}$. There were 44 patients included in the study and the mean follow-up was 10.9 months. There are other case series with very small numbers which suggested capsaicin to have a therapeutic benefit. The positive results of these initial studies have not been borne out in clinical practice, which mirrors the poor results found in several randomised studies of capsaicin therapy in pain ${ }^{[44]}$. Capsaicin at higher doses desensitises the TRPV1 receptor. The doses used in the treatment of pain and itch do not desensitise the receptor at all; like tacrolimus, they probably work by counter-irritation rather than by inhibition of pruritic processing. It remains safe at this dosage, but most patient cease use of capsaicin because of the burning sensation.

\section{Anal tattooing}

Wolloch described the first series of therapeutic methylene blue injection for PA in 1979 in 9 patients with the injection of $15-20 \mathrm{ml}$ of $1 \%$ methylene blue with a good response in all ${ }^{[45]}$. Eusebio described this as anal tattooing by injecting $30 \mathrm{ml}$ of $0.5 \%$ methylene blue ${ }^{[46,47]}$. This study reported complete relief in half of 26 patients, a partial response in $30 \%$, and no response in $9 \%$. The sole use of methylene blue resulted in skin necrosis in some patients. This technique has subsequently being modified to reduce the risk of skin necrosis. There are 4 studies which used a modified admixture are included in Table $1^{[37-40]}$.

Botterill et al., used a mixture of $5 \mathrm{ml}$ of $1 \%$ methylene blue, $15 \mathrm{ml}$ of $1 \%$ Lignocaine and $100 \mathrm{mg}$ of hydrocortisone on 25 patients $^{[34]}$. With the first injection, 16 out of 25 were rendered symptom free. The 9 who were non-responders had a second injection and 6 of these patients also responded. Their median follow-up was 11 months (2 - 25 months). A case series by Samalavicius et al described a technique with admixture of $10 \mathrm{ml}$ of $2 \%$ methylene blue with $5 \mathrm{ml}$ of $2 \%$ lignocaine and $5 \mathrm{ml}$ of saline ${ }^{[39]}$. They had a series of 10 patients all of whom were symptom free at 4 weeks. This study had a follow up of a median of 47 months. Over all $20 \%$ of the patients remained symptom free and a further $40 \%$ had recurrent symptoms of lesser magnitude. A further case series by Sutherland et al used a mixture of $10 \mathrm{ml}$ of $1 \%$ methylene blue, $20 \mathrm{ml}$ of $0.5 \%$ bupivacaine with 1:200000 adrenaline and $1 \mathrm{ml}$ of methyl prednisolone ${ }^{[40]}$. The series had 49 patients of which 30 had complete resolution of symptoms after one injection. The non-responders had a second injection, 4 of which had a complete resolution of symptoms. However, these assessments were made at 8 weeks follow-up. Mentes et al., used an admixture of $15 \mathrm{ml}$ of $2 \%$ methylene blue and $0.5 \%$ lignocaine (variable volume used) in 30 patients $^{[37]}$. Twenty-four of them were symptom free after one injection and 5 patients with partial response received a second injection. Four of these 5 also became symptom free. At 6 month follow up 25 remained symptoms free and 23 remain symptom free at 1 year follow-up.

Methylene blue anal tattooing (Figure 2) seems to be the most effective therapeutic effect on PA symptoms of all the intervention described. However, the admixture concentration of methylene blue varies from $0.25-1 \%$. The type of steroid used also varies. One study by Novak et al suggested that the injectable steroid should be a succinate rather than a phosphate, as the latter formulation produced a burning itch in itself $f^{[42]}$. The mechanism of action of methylene blue is by sensory neurolysis, as suggested in an electron microscopy study showing nerve-ending damage by the methylene blue. This explains the hypoaesthesia induced. However, this sensory neurolysis does recover within a year, which may explain relapse of PA seen with this therapy.

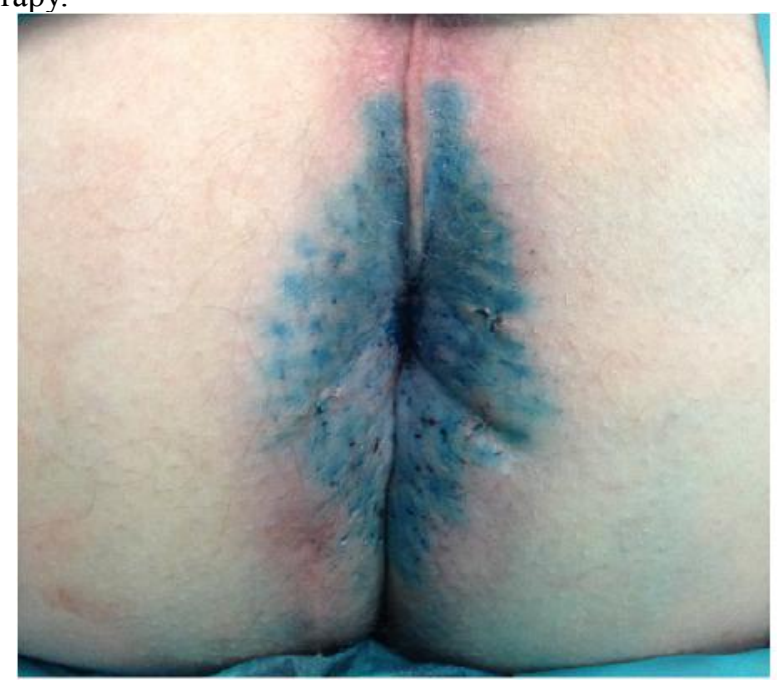




\section{Conclusion}

The aim of this paper was a review of the best evidence available on the current treatment of PA. We set up to perform a systematic review, but were unable due to the heterogeneity of evidence so we performed a review instead. The methodology used in systemic reviews has been followed as closely, without being able to collate the results from different studies. The aetiology, examination and investigations for managing PA patients has not been described in detail and can be found well described elsewhere. There remains an evidence gap in the pathophysiology and treatment of PA. More research is needed, but there are so many unknowns about the nature of PA, this will be currently difficult to perform. The understanding of pruritus and pruritic processing is in its infancy and lags our knowledge of pain. Conservative therapies help with mild to moderate symptoms. Newer therapies such as tacrolimus and capsaicin have failed to live up to initial promise, although they can be effective in a few. Anal tattooing shows the greatest promise.

\section{References}

1. Chaudhry, V., Bastawrous, A. Idiopathic pruritus ani. (2003) Seminars in Colon and Rectal Surgery 14(4): 196-202.

2. Honna, F. Fundamental principles in anal surgery. II. (1965) Shujutsu 19(5): 392-399

3. Open Science Collaboration. Estimating the reproducibility of psychological science. (2015) Science 349(6251): aac4716.

4. Moher, D., Liberati, A., Tetzlaff, J., et al. Preferred reporting items for systematic reviews and meta-analyses: the PRISMA statement. (2009) BMJ 151(4): 264-269.

5. von Elm, E., Altman, D.G., Egger, M., et al. The Strengthening the Reporting of Observational Studies in Epidemiology (STROBE) statement: guidelines for reporting observational studies. (2007) BMJ 335(7624): 806-808.

6. Rothman, S. Physiology of itching. (1941) Physiological reviews 21(2): 357-381.

7. Akiyama, T., Carstens, M.I., Carstens, E. Enhanced scratching evoked by PAR-2 agonist and 5-HT but not histamine in a mouse model of chronic dry skin itch. (2010) Pain 151(2): 378-383.

8. Morita, T., McClain, S.P., Batia, L.M., et al. HTR7 mediates serotonergic acute and chronic itch. (2015) Neuron 87(1): 124-138.

9. Schmelz, M., Hilliges, M., Schmidt, R., et al. Active "itch fibers" in chronic pruritus. (2003) Neurology 61(4): 564-566.

10. Bromage, P.R., Camporesi, E.M., Durant, P.A., et al. Nonrespiratory side effects of epidural morphine. (1982) Anesth Analg 61(6): 490-495. 11. Daniel, G.L., Longo, W.L., Vernava, A.M. Jr. Pruritus ani. Causes and concerns. (1994) Dis Colon Rectum 37(7): 670-674.

12. Smith, L.E., Henrichs, D., McCullah, R.D. Prospective studies on the etiology and treatment of pruritus ani. (1982) Dis Colon Rectum 25(4): 358-363.

13. Bowyer, A., McColl, I. A study of 200 patients with pruritus ani (1970) Proc R Soc Med 63(Suppl 1): 96-98.

14. Dasan, S., Neill, S.M., Donaldson, D.R., et al. Treatment of persistent pruritus ani in a combined colorectal and dermatological clinic. (1999) Br J Surg 86(10): 1337-1340.

15. Zuccati, G., Lotti, T., Mastrolorenzo, A., et al. Pruritus ani. (2005) Dermatologic Ther 18(4): 355-362.

16. Pirone, E., Infantino, A., Masin, A., et al. Can proctological procedures resolve perianal pruritus and mycosis? (1992) Int J Colorectal Dis 7(1):18-20.

17. Caplan, R.M. The irritant role of feces in the genesis of perianal itch. (1966) Gastroenterology 50(1): 19-23.
18. Friend, W.G. The cause and treatment of idiopathic pruritus ani. (1977) Dis Colon Rectum 20(1): 40-42.

19. Harmston, C., Jones, O.M., Cunningham, C., et al. The relationship between internal rectal prolapse and internal anal sphincter function. (2011) Colorectal Dis 13(7): 791-795.

20. Dodi, G., Pirone, E., Bettin, A., et al. The mycotic flora in proctological patients with and without pruritus ani. (1985) Br J Surg 72(12): 967-969.

21. Siddiqi, S., Vijay, V., Ward, M. et al. Pruritus ani. (2008) Ann R Coll Surg Engl 90(6): 457-463.

22. Weichert, G.E. An approach to the treatment of anogenital pruritus. (2004) Dermatol Ther 17(1): 129-133.

23. Alexander-Williams, J. Causes and management of anal irritation. (1983) BMJ 287(6404): 1528.

24. Harrington, C.I., Lewis, F.M., McDonagh, A.J., et al. Dermatological causes of pruritus ani. (1992) BMJ 305: 955.

25. Kligman, A.M., Frosch, P.J. Steroid addiction. (1979) Int J Dermatol 18(1): 23-31.

26. Abu-Asi, M.J., White, I.R., McFadden, J.P., et al. Patch testing is clinically important for patients with perianal dermatoses and pruritus ani. (2016) Contact Dermatitis 74(5): 298-300.

27. Wahlgren, C.F., Hägermark, O., Bergström, R. The antipruritic effect of a sedative and a non-sedative antihistamine in atopic dermatitis. (1990) Br J Dermatol 122(4): 545-551.

28. Collinson, R., Wijffels, N., Cunningham, C., et al. Laparoscopic ventral rectopexy for internal rectal prolapse: short-term functional results. (2010) Colorectal Dis 12(2): 97-104.

29. Al-Ghnaniem, R., Short, K., Pullen, A., et al. 1\% hydrocortisone ointment is an effective treatment of pruritus ani: a pilot randomized controlled crossover trial. (2007) Int J Colorectal Dis 22(12): 14631467.

30. Jensen, S.L. A randomised trial of simple excision of non-specific hypertrophied anal papillae versus expectant management in patients with chronic pruritus ani. (1988) Ann R Coll Surg Engl 70(6): 348.

31. Lysy, J., Sistiery-Ittah, M., Israelit, Y., et al. Topical capsaicin-a novel and effective treatment for idiopathic intractable pruritus ani: a randomised, placebo controlled, crossover study. (2003) Gut 52(9): $1323-1326$

32. Suys, E. Randomized study of topical tacrolimus ointment as possible treatment for resistant idiopathic pruritus ani. (2012) J Am Acad Dermatol 66(2): 327-328

33. Ucak, H., Demir, B., Cicek, D., et al. Efficacy of topical tacrolimus for the treatment of persistent pruritus ani in patients with atopic dermatitis. (2013) J Dermatolog Treat 24(6): 454-457.

34. Botterill, I.D., Sagar, P.M. Intra-dermal methylene blue, hydrocortisone and lignocaine for chronic, intractable pruritus ani. (2002) Colorectal Dis 4(2): 144-146.

35. Gupta, P.J. A study of the symptomatology of hypertrophied anal papillae and fibrous anal polyps. (2005) Bratisl Lek Listy 106(1): 3033.

36. Kang, G., Kim, B., Kim, J., et al. Prospective study on the treatment of intractable pruritus ani with Triamcinolone local injection. (2009) Dis Colon Rectum 52(4): 857-857.

37. Mentes, B.B., Akin, M., Leventoglu, S., et al. Intradermal methylene blue injection for the treatment of intractable idiopathic pruritus ani: results of 30 cases. (2004) Tech Coloproctol 8(1): 11-14.

38. Miravalle, O.R., Vazquez, F.D., Bolino, C., et al. Sa2041 Response to a Therapeutic Algorithm and Short Term Follow up of a Poorly Managed Symptom: Idiopathic Pruritus Ani. (2013) Gastroenterology 144(5): S-367.

39. Samalavicius, N.E., Poskus, T., Gupta, R.K., et al. Long-term results of single intradermal $1 \%$ methylene blue injection for intractable idiopathic pruritus ani: a prospective study. (2012) Tech Coloproctol 16(4): 295-299.

40. Sutherland, A.D., Faragher, I.G., Frizelle, F.A. Intradermal injection of methylene blue for the treatment of refractory pruritus ani. (2009) Colorectal Dis 11(3): 282-287. 
41. Xie, Z., Lan, Y. Effect and molecule psychophysical mechanism analysis of tacrolimus ointment in treating male patients with pruritus ani. (2014) J Invest Dermatol 134: S100-S100.

42. Novak, E., Gilbertson, T.J., Seckman, C.E. Anorectal pruritus after intravenous hydrocortisone sodium succinate and sodium phosphate. (1976) Clin Pharmacol Ther 20(1): 109-112.

43. Oztaş, M.O., Oztaş, P., Onder, M. Idiopathic perianal pruritus: washing compared with topical corticosteroids. (2004) Postgrad Med J 80(943): 295-297.

44. Sokołowski P. Capsaicin in pain therapy. (1994) Wiadomości lekarskie (Warsaw, Poland: 1960) 47(13-14): 527-532.

45. Wolloch, Y., Dintsman, M. A simple and effective method of treatment for intractable pruritus ani. (1978) Am J Proctol Gastroenterol Colon Rectal Surg 30(1): 34-36.

46. Eusebio, E.B., Graham, J., Mody, N. Treatment of intractable pruritus ani. (1990) Dis Colon Rectum 33(9): 770-772.

47. Eusebio, E.B. New treatment of intractable pruritus ani. (1991) Dis Colon Rectum 34(3): 289-289. 